\title{
A COMPARATIVE STUDY BETWEEN TWO SYSTEMS WITH AND WITHOUT AWARENESS IN CONTROLLING HIV/AIDS
}

\author{
ShUBHANKAR SAHA ${ }^{a}$, Priti KUMAR ROY ${ }^{a, *}$ \\ ${ }^{a}$ Centre for Mathematical Biology and Ecology, Department of Mathematics \\ Jadavpur University, Kolkata 700032, India \\ e-mail: pritiju@gmail.com
}

\begin{abstract}
It has always been a priority for all nations to reduce new HIV infections by implementing a comprehensive HIV prevention programme at a sufficient scale. Recently, the 'HIV counselling \& testing' (HCT) campaign is gaining public attention, where HIV patients are identified through screening and immediately sent under a course of antiretroviral treatment (ART), neglecting the time extent they have been infected. In this article, we study a nonlinear mathematical model for the transmission dynamics of HIV/AIDS system receiving drug treatment along with effective awareness programs through media. Here, we consider two different circumstances: when treatment is only effective and when both treatment and awareness are included. The model is analyzed qualitatively using the stability theory of differential equations. The global stabilities of the equilibria under certain conditions are determined in terms of the model reproduction number. The effects of changes in some key epidemiological parameters are investigated. Projections are made to predict the long term dynamics of the disease. The epidemiological implications of such projections on public health planning and management are discussed. These studies show that the aware populations were less vulnerable to HIV infection than the unaware population.
\end{abstract}

Keywords: epidemic model, HIV, awareness, anti-retroviral therapy, basic reproductive number, numerical simulation.

\section{Introduction}

HIV/AIDS continues to be a major public health issue that disproportionately affects our human society. According to $\mathrm{WHO}$, there were approximately 34 million people worldwide living with HIV/AIDS in 2014 and 36.7 million in 2015, meaning that the HIV cases increased by $7.94 \%$ globally in one year from the beginning of 2014 to the end of 2015. WHO also reported that in the same time period (2014-2015), the global coverage of antiretroviral therapy ranged from $42 \%$ to $46 \%$. Therefore, the fact is that, although increasing emphasis is being placed and people are under coverage of antiretroviral treatment, no indicatory outcomes have been seen in positive prevention of HIV (UDAIDS/WHO, 2014; UDAIDS/WHO, 2015). One of the most significant reasons for this failure was found as the 'lack of awareness' among people. Thus, developing awareness levels among masses, especially in the high risk infected category, is very important.

The HCT program is a scheme which directly takes part in both 'HIV prevention' (through the knowledge

*Corresponding author of one's HIV status) and 'treatment of HIV infection' (through knowing what to do in case one is positive). Extensive research has been done based on the disease dynamics with combinational drug therapy or using other strategies, but continuing drug treatment along with simultaneous awareness programs through media is a novel conception which should be taken in our consideration. The major question we ask here is: How does such an initiative influence the course of HIV/AIDS epidemic? In this paper we develop a mathematical model that incorporates this intervention with the intention of assessing the effectiveness of HCT and reduction in HIV transmission due to treatment initiation.

Mathematical models, their analysis and results have been used broadly in research into the epidemiology of HIV/AIDS to improve our understanding of major contributing factors to the pandemic. Incorporation of interventions in these models has attracted significant attention in recent years (Gumel et al., 2006; Roy, 2015; Nyabadza, 2006; Cai et al., 2009; Elbasha and Gumel, 2006; Chatterjee et al., 2015). The epidemiology of HIV/AIDS has moved beyond the virus and the risk 
factors associated with its transmission to a more detailed observation of the mechanisms associated with the spread, distribution and impact of interventions on the population. Consequently, the impact of health policies, such as poor access to care, delayed treatment or the use of screening for asymptomatic cases can be evaluated.

Chatterjee and Roy (2012) formulated a basic mathematical model to study the cellular infection for HIV positive patients within the human immune system. They showed that the controlled drug therapy in different cases gives better understanding to control the diseases progression or to get rid of the disease. In the work of Tripathi et al. (2007), a model that considers infection leading to asymptomatic HIV infectives who are later screened and finally develop AIDS is presented without interventions. A similar approach was considered earlier by Hyman et al. (2003) with differential infectivity and staged progression models. Al-arydah and Smith (2015) considered a model where they investigated how testing, educating HIV-positive cases, treatment and drug resistance affects the HIV epidemic. Kiss et al. (2010) proposed a model for a totally aware closed population where the individuals are discriminated according to their response towards the information available. As a result, the spread of infection is controlled, leading to a reduction in the number of individuals becoming infected. Recently, Roy et al. (2015) formulated a model and studied the effect of awareness programs through media on the HIV disease dynamics in a variable population with immigration.

In this present paper, we analyze an epidemic model that provides HCT programs and also awareness related programs through media campaigns in a variable population with immigration. But these results will fall into the non-network epidemic models category. We also investigate a sub-case in Section 3, where it is assumed that no HCT programs are provided and only 'screening \& testing' is available for the whole population. Lastly, in Section 5, we compare both cases numerically to find which system is more feasible and which factors have significant roles to reduce the disease prevalence.

\section{Model}

2.1. System description. The model describes the spread of HIV/AIDS in a high risk population. The total sexually active population at time $t$, denoted by $(N(t))$, is subdivided into six classes that are: unaware susceptibles $(S(t))$, aware susceptibles $\left(S_{+}(t)\right)$, unaware and untreated HIV infected individuals $(I(t))$, diagnosed and treated infected individuals who have not yet developed to AIDS $\left(I_{D}(t)\right)$, diagnosed and treated individuals with clinical $\operatorname{AIDS}\left(I_{D A}(t)\right)$ and aware infected individuals $\left(I_{+}(t)\right)$. Therefore, $N=S+S_{+}+I+I_{D}+I_{D A}+I_{+}$. $\Pi$ is the constant recruitment rate in the susceptible population either by birth or immigration and $d$ is the natural death rate. $d_{I}, \mu_{I}, \mu_{A}$ and $\mu_{+}$are the additional death rates for $I, I_{D}, I_{D A}$ and $I_{+}$, respectively. The force of infection $\lambda$ is given by

$$
\lambda=\beta \frac{I+\lambda_{i \tau} I_{D}+\lambda_{i \tau a} I_{D A}+\lambda_{+} I_{+}}{N},
$$

where $\beta$ is the product of the effective contact rate between susceptible and infected individuals that is sufficient to result in HIV infection and the transmission probability of HIV per contact; $\delta_{\tau}=\delta \cdot \tau$ is the rate at which people are diagnosed and treated $(\delta=$ diagnosis rate and $\tau=$ treatment rate); $p$ is the proportion of diagnosed individuals who have not yet developed to AIDS $(0 \leq p \leq 1) ; \nu$ is the rate of progression from HIV diagnosis to the AIDS class. Susceptible individuals transfer from the unaware to the aware class at a rate $c$ and infected individuals move to the aware class at a rate $\sigma_{x \in E}, E=\{i, i \tau, i \tau a\}$. Note that we ignore the interactions between aware individuals and HIV infected subcases, because it is assumed that once a person (with or without AIDS) becomes aware, he/she will avoid being in contact with the infective and does not get involved in any sexual relations or in any other means that causes AIDS. But due to the lack of their memory, a portion will transfer to the unaware class from the aware population. We assume that $\theta_{1}$ and $\theta_{2}$ are the transfer rates from aware to unaware for susceptible and infective cases, respectively. The population movements between

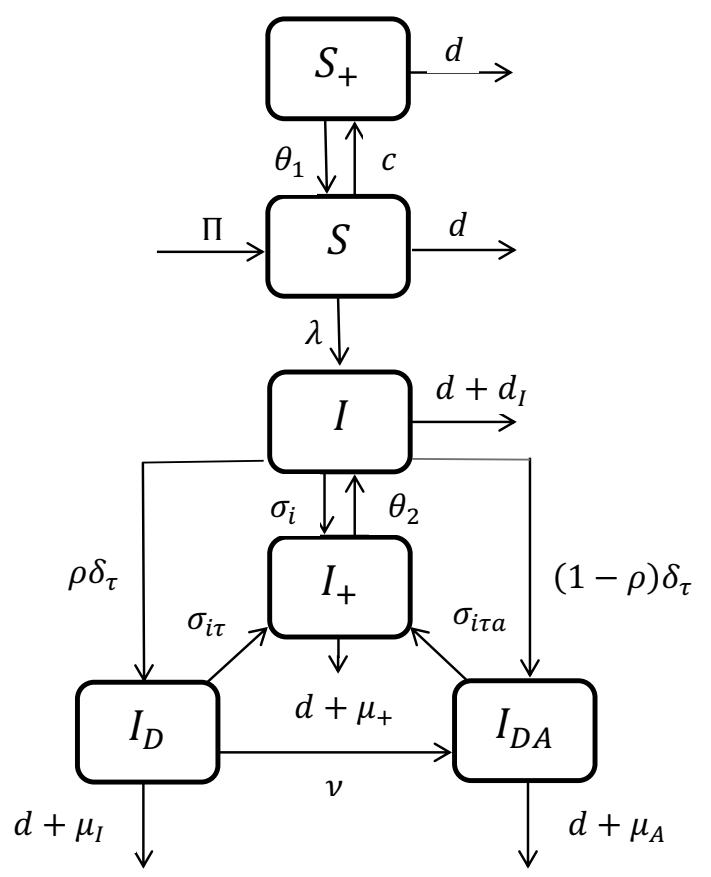

Fig. 1. Flow diagram of the system (2). 
different compartments are described in Fig. 1.

On the basis of the above assumptions, the dynamics of the model are governed by the following system of nonlinear ordinary differential equations:

$$
\begin{aligned}
\frac{\mathrm{d} S}{\mathrm{~d} t}= & \Pi-(\lambda+d+c) S+\theta_{1} S_{+}, \\
\frac{\mathrm{d} S_{+}}{\mathrm{d} t}= & c S-\left(d+\theta_{1}\right) S_{+}, \\
\frac{\mathrm{d} I}{\mathrm{~d} t}= & \lambda S-\left(d+d_{I}+\delta_{\tau}+\sigma_{i}\right) I+\theta_{2} I_{+}, \\
\frac{\mathrm{d} I_{D}}{\mathrm{~d} t}= & p \delta_{\tau} I-\left(d+\mu_{I}+\nu+\sigma_{i \tau}\right) I_{D}, \\
\frac{\mathrm{d} I_{D A}}{\mathrm{~d} t}= & (1-p) \delta_{\tau} I+\nu I_{D}-\left(d+\mu_{A}\right. \\
& \left.+\sigma_{i \tau a}\right) I_{D A}, \\
\frac{\mathrm{d} I_{+}}{\mathrm{d} t}= & \sigma_{i} I+\sigma_{i \tau} I_{D}+\sigma_{i \tau a} I_{D A}-\left(d+\theta_{2}\right. \\
& \left.+\mu_{+}\right) I_{+}
\end{aligned}
$$

with the positive initial conditions given by

$$
\begin{array}{r}
S(0)=S_{0}, \quad S_{+}(0)=S_{+_{0}}, \quad I(0)=I_{0}, \\
I_{D}(0)=I_{D_{0}}, \quad I_{D A}(0)=I_{D A_{0}}, \quad I_{+}(0)=I_{+_{0}} .
\end{array}
$$

Since the above system represents population dynamics, it is assumed that all the state variables are non-negative at time $t=0$ with $I+\lambda_{i \tau} I_{D}+\lambda_{i \tau a} I_{D A}+\lambda_{+} I_{+}>0$ and all other parameters of the model are non-negative.

2.2. Model properties. System (2) will be analyzed in a domain $\mathcal{D} \subset \mathbb{R}_{+}^{6}$, where all feasible solutions enter the region

$\mathcal{D}=\left\{\left(S, S_{+}, I, I_{D}, I_{D A}, I_{+}\right) \in \mathbb{R}_{+}^{6}: 0 \leq N \leq \frac{\Pi}{d}\right\}$.

Theorem 1. The solutions of system (2) with initial conditions (3) satisfy $S(t)>0, S_{+}(t)>0, I(t)>$ $0, I_{D}(t)>0, I_{D A}(t)>0, I_{+}(t)>0$ for all $t>0$. The region $\mathcal{D} \in \mathbb{R}_{+}^{6}$ is positively invariant and attracting with respect to system (2).

Proof. From the first equation of (2) we have

$$
\dot{S} \geq-(\lambda+d+c) S,
$$

giving

$$
S(t) \geq S(0) \exp \left(-\int_{0}^{t}(\lambda(s)+d+c) \mathrm{d} s\right)>0 .
$$

In a similar fashion we can show that $S_{+}(t), I(t), I_{D}(t), I_{D A}(t)$ and $I_{+}(t)$ are all strictly positive. Thus we can conclude that all solutions of system (2) remain positive for all $t>0$.
Now, we will show that all feasible solutions are uniformly bounded in $\mathcal{D}$. Using the fact that $N=S+$ $S_{+}+I+I_{D}+I_{D A}+I_{+}$, we get

$$
\begin{aligned}
\dot{N} & =\Pi-d N-d_{I} I-\mu_{I} I_{D}-\mu_{A} I_{D A}-\mu_{+} I_{+} \\
& \leq \Pi-d N .
\end{aligned}
$$

Solving this differential equation, we have

$$
0 \leq N(t) \leq \frac{\Pi}{d}+N(0) e^{-d t},
$$

where $N(0)$ represents the initial value of the total population at time $t=0$. Thus, as $t \rightarrow \infty, 0 \leq N \leq \Pi / d$. Therefore, it it clear from the above that $\Pi / d$ is an upper bound to $N$, provided $N(0) \leq \Pi / d$. If $N(0)>\Pi / d$, then $N$ will decrease to this level. Thus, all feasible solutions of the system enter or remain in the region $\mathcal{D}$. Further, the existence, uniqueness and continuation results hold for the system in this region.

\section{Sub-model analysis}

In this section, we will examine the case when no HCT programs are provided and only screening and testing is available for the whole population, i.e.,

$$
\begin{aligned}
\frac{\mathrm{d} S}{\mathrm{~d} t} & =\Pi-(\lambda+d) S, \\
\frac{\mathrm{d} I}{\mathrm{~d} t} & =\lambda S-\left(d+d_{I}+\delta_{\tau}\right) I, \\
\frac{\mathrm{d} I_{D}}{\mathrm{~d} t} & =p \delta_{\tau} I-\left(d+\mu_{I}+\nu\right) I_{D}, \\
\frac{\mathrm{d} I_{D A}}{\mathrm{~d} t} & =(1-p) \delta_{\tau} I+\nu I_{D}-\left(d+\mu_{A}\right) I_{D A},
\end{aligned}
$$

where $\lambda=\beta\left(I+\lambda_{i \tau} I_{D}+\lambda_{i \tau a} I_{D A}\right) / N$ and $N=S+$ $I+I_{D}+I_{D A}$.

3.1. Disease-free equilibrium (DFE) and the reproductive number. The sub-model (5) has a disease-free equilibrium, obtained by setting right-hand sides of system (5) to zero, given by

$$
E_{0}=\left(\bar{S}, \bar{I}, \bar{I}_{D}, \bar{I}_{D A}\right)=(\Pi / d, 0,0,0) .
$$

The basic reproductive number can be established by using the next generation operator (Van den Driessche and Watmough, 2002). Using this approach, we have

$$
\mathcal{F}=\left[\begin{array}{c}
0 \\
\lambda S \\
0 \\
0
\end{array}\right]
$$

and

$$
\mathcal{V}=\left[\begin{array}{c}
(\lambda+d) S-\Pi \\
\left(d+d_{I}+\delta_{\tau}\right) I \\
-p \delta_{\tau} I+\left(d+\mu_{I}+\nu\right) I_{D} \\
-(1-p) \delta_{\tau} I-\nu I_{D}+\left(d+\mu_{A}\right) I_{D A}
\end{array}\right]
$$


The infected compartments are $I, I_{D}$ and $I_{D A}$. Thus the matrices $F$ and $V$ for the new infection terms and the remaining transfer terms are given by

$$
\begin{gathered}
F=\left[\begin{array}{ccc}
\beta & \beta \lambda_{i \tau} & \beta \lambda_{i \tau a} \\
0 & 0 & 0 \\
0 & 0 & 0
\end{array}\right], \\
V=\left[\begin{array}{ccc}
\left(d+d_{I}+\delta_{\tau}\right) & 0 & 0 \\
-p \delta_{\tau} & \left(d+\mu_{I}+\nu\right) & 0 \\
-(1-p) \delta_{\tau} & -\nu & \left(d+\mu_{A}\right)
\end{array}\right] .
\end{gathered}
$$

Therefore, the model reproductive number is given by

$$
\begin{aligned}
R_{0}= & \rho\left(F V^{-1}\right) \\
= & \beta\left[\frac{1}{\left(d+d_{I}+\delta_{\tau}\right)}+\frac{\lambda_{i \tau} p \delta_{\tau}}{\left(d+d_{I}+\delta_{\tau}\right)\left(d+\mu_{I}+\nu\right)}\right. \\
& \left.+\frac{\delta_{\tau} \lambda_{i \tau a}\left(\nu+(1-p)\left(d+\mu_{I}\right)\right)}{\left(d+d_{I}+\delta_{\tau}\right)\left(d+\nu+\mu_{I}\right)\left(d+\mu_{A}\right)}\right],
\end{aligned}
$$

the spectral radius of the next generation matrix $F V^{-1}$.

Thus, we have proved the following theorem on the local stability of $E_{0}$.

Theorem 2. The disease-free equilibrium $E_{0}$ for the system (5) is locally asymptotically stable whenever $R_{0}<$ 1 and unstable otherwise.

Remark 1. It is to be noted that if $\lambda_{i \tau} p\left(d+d_{I}\right)(d+$ $\left.\mu_{A}\right)+\lambda_{i \tau a}\left[\nu(1-p)\left(d+\mu_{I}\right)\right]<d+\mu_{I}+\nu$, then

$$
\frac{\partial R_{0}}{\partial \delta_{\tau}}<0
$$

This implies that as long as $\lambda_{i \tau} p\left(d+d_{I}\right)\left(d+\mu_{A}\right)+$ $\lambda_{i \tau a}\left[\nu(1-p)\left(d+\mu_{I}\right)\right]<d+\mu_{I}+\nu$, antiretroviral treatment will have a significant input in HIV/AIDS control.

3.1.1. Global stability of $\boldsymbol{E}_{\mathbf{0}}$. We now use Theorem 1 of Castillo-Chavez et al. (2002) to show the global stability of the DFE for the system (5), which can be stated as described in the following theorem.

Theorem 3. Rewrite system (5) in the form

$$
\begin{aligned}
X^{\prime}(t) & =H(X, Y), \\
Y^{\prime}(t) & =G(X, Y), \quad G(X, Y)=0
\end{aligned}
$$

where $X=(S)$ and $Y=\left(I, I_{D}, I_{D A}\right)$ with $X \in \mathbb{R}_{+}$denoting (its component) the number of uninfected individual and $Y \in \mathbb{R}_{+}^{3}$ denoting (its components) the number of infected individuals. Assume that following conditions are satisfied:

(H1) For $X^{\prime}(t)=H\left(X^{*}, 0\right), X^{*}$ is globally asymptotically stable,

$$
\begin{aligned}
& G(X, Y)=A Y-\widehat{G}(X, Y), \widehat{G}(X, Y) \geq 0 \\
& \text { for }(X, Y) \in \mathcal{D} .
\end{aligned}
$$

Then the fixed point $E_{0}=\left(X_{0}, 0\right)$, where $X_{0}=(\Pi / d)$, is a globally asymptotically stable point of model system (5), provided $R_{0}<1$.

We consider $H(X, 0)=[\Pi-d X]$,

$$
A=\left[\begin{array}{cc}
\beta-\left(d+d_{I}+\delta_{\tau}\right) & \\
p \delta_{\tau} & \\
(1-p) \delta_{\tau} & \\
\beta \lambda_{i \tau} & \beta \lambda_{i \tau a} \\
-\left(d+\mu_{I}+\nu\right) & 0 \\
\nu & -\left(d+\mu_{A}\right)
\end{array}\right]
$$

$\widehat{G}(X, Y)=\left[\begin{array}{c}\beta\left(I+\lambda_{i \tau} I_{D}+\lambda_{i \tau a} I_{D A}\right)(1-S / N) \\ 0 \\ 0\end{array}\right]$.

Therefore,

$$
\widehat{G}(X, Y) \geq 0
$$

for all

$$
(X, Y) \in\left\{\left(I, I_{D}, I_{D A}\right) \in \mathbb{R}_{+}^{3}: N \leq \frac{\Pi}{d}\right\} .
$$

As a result, the conditions of Theorem 1 of Castillo-Chavez et al. (2002) are satisfied, and we can conclude the result in the next theorem.

Theorem 4. The disease-free equilibrium $E_{0}$ for system (5) is globally asymptotically stable whenever $R_{0}<1$.

3.2. Endemic equilibrium point (EE). The endemic equilibrium point of the system (5) is given by $E^{*}\left(S^{*}, I^{*}, I_{D}^{*}, I_{D A}^{*}\right)$, where

$$
\begin{aligned}
S^{*} & =\frac{\Pi}{\lambda^{*}+d}, \\
I^{*} & =\frac{\Pi \lambda^{*}}{\left(\lambda^{*}+d\right)\left(d+d_{I}+\delta_{\tau}\right)}, \\
I_{D}^{*} & =\frac{\Pi \rho \delta_{\tau} \lambda^{*}}{\left(\lambda^{*}+d\right)\left(d+d_{I}+\delta_{\tau}\right)\left(d+\mu_{I}+\nu\right)}, \\
I_{D A}^{*} & =\frac{\left.\Pi \delta_{\tau} \lambda^{*}\left[(1-p)\left(d+\mu_{I}+\nu\right)\right)+p \nu\right]}{\left(\lambda^{*}+d\right)\left(d+\mu_{A}\right)\left(d+d_{I}+\delta_{\tau}\right)\left(d+\mu_{I}+\nu\right)}, \\
N^{*} & =\frac{\Pi\left[1+\lambda^{*} \psi\right]}{\lambda^{*}+d},
\end{aligned}
$$


with

$$
\begin{aligned}
\psi= & \frac{1}{\left(d+d_{I}+\delta_{\tau}\right)}+\frac{p \delta_{\tau}}{\left(d+d_{I}+\delta_{\tau}\right)\left(d+\mu_{I}+\nu\right)} \\
& +\frac{\delta_{\tau}\left[(1-p)\left(d+\mu_{I}+\nu\right)+p \nu\right]}{\left(d+d_{I}+\delta_{\tau}\right)\left(d+\mu_{I}+\nu\right)\left(d+\mu_{A}\right)} .
\end{aligned}
$$

Substituting $S^{*}, I^{*}, I_{D}^{*}$ and $I_{D A}^{*}$ into the equation $\lambda=\beta\left(I+\lambda_{i \tau} I_{D}+\lambda_{i \tau a} I_{D A}\right) / N$, we obtain a quadratic polynomial whose roots are $\lambda^{*}=0$ and $\lambda^{*}=$ $\left(R_{0}-1\right) / \psi$. The case $\lambda^{*}=0$ gives the disease free equilibrium discussed in Section 3.1.

Note that $R_{0}>1$ implies $\lambda^{*}>0$. Thus, we can conclude the following result.

Theorem 5. Whenever $R_{0}>1$, the disease-free equilibrium (DFE) $E_{0}$ becomes unstable and the endemic equilibrium point (EE) exists.

3.2.1. Local stability of the EE. The variational matrix around the endemic equilibrium point $E^{*}$ is

$$
J\left(E^{*}\right)=\left[\begin{array}{cccc}
-m_{11} & -m_{12} & -m_{13} & -m_{14} \\
m_{21} & m_{22} & m_{23} & m_{24} \\
0 & m_{32} & -m_{33} & 0 \\
0 & m_{42} & m_{43} & -m_{44}
\end{array}\right]
$$

with $m_{11}=d+\lambda^{*}, m_{12}=\beta S^{*} / N^{*}, m_{13}=m_{23}=$ $\beta \lambda_{i \tau} S^{*} / N^{*}, m_{14}=m_{24}=\beta \lambda_{i \tau a} S^{*} / N^{*}, m_{22}=$ $\beta S^{*} / N^{*}-\left(d+d_{I}+\delta_{\tau}\right), m_{32}=p \delta_{\tau}, m_{33}=$ $\left(d+\mu_{I}+\nu\right), m_{42}=(1-p) \delta_{\tau}, m_{43}=\nu$ and $m_{44}=d+\mu_{A}$.

The characteristic equation corresponding to the above matrix is

$$
\varrho^{4}+A_{1} \varrho^{3}+A_{2} \varrho^{2}+A_{3} \varrho+A_{4}=0,
$$

where

$$
\begin{aligned}
A_{1}= & m_{11}-m_{22}+m_{33}+m_{44}, \\
A_{2}= & m_{33} m_{44}+m_{12} m_{21}-\left(m_{22} m_{33}+m_{22} m_{44}\right. \\
& \left.+m_{23} m_{32}+m_{24} m_{42}\right), \\
A_{3}= & m_{21}^{2} m_{33}+m_{21}^{2} m_{44}+m_{21} m_{13} m_{32} \\
& +m_{21} m_{14} m_{42}-\left(m_{22} m_{33} m_{44}+m_{23} m_{32} m_{44}\right. \\
& \left.+m_{24} m_{32} m_{43}+m_{24} m_{42} m_{33}\right), \\
A_{4}= & m_{12} m_{21} m_{33} m_{44}+m_{21} m_{13} m_{32} m_{44} \\
& +m_{21} m_{14} m_{32} m_{43}+m_{21} m_{14} m_{42} m_{33} \\
& -\left(m_{11} m_{22} m_{33} m_{44}+m_{11} m_{23} m_{32} m_{44}\right. \\
& \left.+m_{11} m_{24} m_{32} m_{43}+m_{11} m_{24} m_{42} m_{33}\right) .
\end{aligned}
$$

Thus, using the Routh-Hurwitz criterion, we can conclude the following result.

Theorem 6. The system (5) around the endemic equilibrium $E^{*}$ is locally asymptotically stable if $A_{1}>0, A_{3}>$ $0, A_{4}>0$ and $A_{1} A_{2} A_{3}>A_{3}^{2}+A_{1}^{2} A_{4}$.
3.2.2. Global stability of the EE. To prove the global stability of the endemic equilibrium, we assume that the population does not change significantly over time and the relation $\Pi=d N+d_{I} I+\mu_{I} I_{D}+\mu_{A} I_{D A}$ holds. While this may not be true for the case of South Africa at the present moment, evidence of a declining population growth due to HIV/AIDS mortality has been observed (Statistics, 2006) and a stagnation of the population growth is forecasted. This allows the force of infection to be driven by a normalized mass action incidence function. To attain the full characterization of endemic equilibrium, we construct a Lyapunov function and the results thus found are stated in the following theorem whose proof is given in Appendix A

Theorem 7. The endemic equilibrium $E^{*}$ is globally asymptotically stable if $\Pi=d N+d_{I} I+\mu_{I} I_{D}+\mu_{A} I_{D A}$ and $R_{0}>1$.

Therefore, from the above discussion we have found the existence conditions for both the equilibrium states: the disease-free state and the endemic state. The value of the basic reproduction number and the critical rate of transmission are calculated. We have also proved the local and global stabilities for the disease-free equilibrium and the global stability for the endemic equilibrium. In the next section, we will analyze our complete model system (2).

\section{Full model analysis}

4.1. Disease-free equilibrium $\left(E_{0}^{a}\right)$ and the reproductive ratio $\left(R_{0}^{a}\right)$. System (2) has a disease-free equilibrium obtained by setting the right-hand sides of the equations of system (2) to zero, given by

$$
\begin{aligned}
& E_{0}^{a}: \quad\left(S^{0}, S_{+}^{0}, I^{0}, I_{\tau}^{0}, I_{D A}^{0}, I_{+}^{0}\right) \\
& \quad=\left(\frac{\Pi\left(d+\theta_{1}\right)}{d\left(d+\theta_{1}+c\right)}, \frac{\Pi c}{d\left(d+\theta_{1}+c\right)}, 0,0,0,0\right) .
\end{aligned}
$$

To find the basic reproductive number, the previous technique is used here (applying Van den Driessche and Watmough (2002)). The matrices for the new infection and transition terms are represented by

$$
\begin{gathered}
F_{1}=\left[\begin{array}{cccc}
\beta & \beta \lambda_{i \tau} & \beta \lambda_{i \tau a} & \beta \lambda_{+} \\
0 & 0 & 0 & 0 \\
0 & 0 & 0 & 0 \\
0 & 0 & 0 & 0
\end{array}\right], \\
V_{1}=\left[\begin{array}{cccc}
P_{1} & 0 & 0 & -\theta_{2} \\
-p \delta_{\tau} & P_{2} & 0 & 0 \\
-(1-p) \delta_{\tau} & -\nu & P_{3} & 0 \\
-\sigma_{i} & -\sigma_{i \tau} & -\sigma_{i \tau a} & P_{4}
\end{array}\right],
\end{gathered}
$$


where $P_{1}=\left(d+d_{I}+\delta_{\tau}+\sigma_{i}\right), P_{2}=\left(d+\mu_{I}+\nu+\right.$ $\left.\sigma_{i \tau}\right), P_{3}=\left(d+\mu_{A}+\sigma_{i \tau a}\right), P_{4}=\left(d+\mu_{+}+\theta_{2}\right)$.

Therefore, the reproductive number, $R_{0}^{a}$, for model system (2) is given by

$$
R_{0}^{a}=\beta \frac{1+\lambda_{i \tau} Q_{1}+\lambda_{i \tau a} Q_{2}+\lambda_{+} Q_{3}}{P_{1}+\theta_{2} Q_{3}},
$$

where

$$
\begin{aligned}
Q_{1} & =p \delta_{\tau} / P_{2} \\
Q_{2} & =\left[(1-p) P_{2}+\nu p\right] \delta_{\tau} / P_{2} P_{3} \\
Q_{3} & =\left[\sigma_{i}+\sigma_{i \tau} Q_{1}+\sigma_{i \tau a} Q_{2}\right] / P_{4} .
\end{aligned}
$$

This implies the following result.

Theorem 8. The disease-free equilibrium $E_{0}^{a}$ for the system (2), is locally asymptotically stable whenever

$$
R_{0}^{a}=\beta \frac{1+\lambda_{i \tau} Q_{1}+\lambda_{i \tau a} Q_{2}+\lambda_{+} Q_{3}}{P_{1}+\theta_{2} Q_{3}}<1
$$

and unstable if

$$
R_{0}^{a}=\beta \frac{1+\lambda_{i \tau} Q_{1}+\lambda_{i \tau a} Q_{2}+\lambda_{+} Q_{3}}{P_{1}+\theta_{2} Q_{3}}>1 .
$$

4.2. Endemic equilibrium point (EE). We now analyze the endemic equilibrium of model (2). The endemic equilibrium $E^{* *}\left(S^{* *}, S_{+}^{* *}, I^{* *}, I_{D}^{* *}, I_{D A}^{* *}, I_{+}^{* *}\right)$ of model (2) can be obtained as follows:

$$
\begin{aligned}
S^{* *} & =\frac{\Pi\left(d+\theta_{1}\right)}{\lambda^{* *}\left(d+\theta_{1}\right)+d\left(d+c+\theta_{1}\right)}, \\
S_{+}^{* *} & =\frac{c S^{* *}}{d+\theta_{1}}, \\
I^{* *} & =\frac{\lambda^{* *} S^{* *}}{\left(P_{1}+\theta_{2} Q_{3}\right)}, \\
I_{D}^{* *} & =Q_{1} I^{* *} \\
I_{D A}^{* *} & =Q_{2} I^{* *} \\
I_{+}^{* *} & =Q_{3} I^{* *}, \\
N^{* *} & =S^{* *}+S_{+}^{* *}+I^{* *}+I_{D}^{* *}+I_{D A}^{* *}+I_{+}^{* *} .
\end{aligned}
$$

Substituting (10) in (1), we get a quadratic equation in $\lambda^{* *}$, given by

$$
\lambda^{* *}\left[\lambda^{* *}-\frac{\beta\left(d+\theta_{1}+c\right)}{\left(d+\theta_{1}\right)\left[R_{0}^{a}-\beta \frac{\left(1+Q_{1}+Q_{2}+Q_{3}\right)}{\left(P_{1}+\theta_{2} Q_{3}\right)}\right]}\right]=0 .
$$

The case $\lambda^{* *}=0$ yields the disease free equilibrium, discussed in Section 4.1. For the second solution, $R_{0}^{a}>$ $\beta\left(1+Q_{1}+Q_{2}+Q_{3}\right) /\left(P_{1}+\theta_{2} Q_{3}\right)$ implies $\lambda^{* *}>0$. Therefore, we can conclude the following result.
Theorem 9. The endemic equilibrium for system (2) exists if

$$
R_{0}^{a}>\max \left\{1, \frac{\beta\left(1+Q_{1}+Q_{2}+Q_{3}\right)}{\left(P_{1}+\theta_{2} Q_{3}\right)}\right\}
$$

4.2.1. Local stability of the $\mathbf{E E}\left(E^{* *}\right)$. For the local stability test of the $E E$ of system (2), we will utilize centre manifold theory as described by Castillo-Chavez and Song (2004). In order to apply the centre manifold theory, we make the following changes of variables:

$$
\begin{aligned}
S=x_{1}, \quad S_{+} & =x_{2}, \quad I=x_{3}, \quad I_{D}=x_{4}, \\
I_{D A} & =x_{5}, \quad I_{+}=x_{6},
\end{aligned}
$$

so that

$$
N=\sum_{n=1}^{6} x_{n}
$$

Thus, system (2) can be written as

$$
\begin{aligned}
\frac{\mathrm{d} x_{1}}{\mathrm{~d} t}= & f_{1}=\Pi-(\lambda+d+c) x_{1}+\theta_{1} x_{2}, \\
\frac{\mathrm{d} x_{2}}{\mathrm{~d} t}= & f_{2}=c x_{1}-\left(d+\theta_{1}\right) x_{2}, \\
\frac{\mathrm{d} x_{3}}{\mathrm{~d} t}= & f_{3}=\lambda x_{1}-\left(d+d_{I}+\delta_{\tau}+\sigma_{i}\right) x_{3} \\
& +\theta_{2} x_{6} \\
\frac{\mathrm{d} x_{4}}{\mathrm{~d} t}= & f_{4}=p \delta_{\tau} x_{3}-\left(d+\mu_{I}+\nu+\sigma_{i \tau}\right) x_{4}, \\
\frac{\mathrm{d} x_{5}}{\mathrm{~d} t}= & f_{5}=(1-p) \delta_{\tau} x_{3}+\nu x_{4}-\left(d+\mu_{A},\right. \\
& \left.+\sigma_{i \tau a}\right) x_{5} \\
\frac{\mathrm{d} x_{6}}{\mathrm{~d} t}= & f_{6}=\sigma_{i} x_{3}+\sigma_{i \tau} x_{4}+\sigma_{i \tau a} x_{5} \\
& -\left(d+\theta_{2}+\mu_{+}\right) x_{6}
\end{aligned}
$$

where

$$
\lambda=\frac{\beta}{N}\left(x_{3}+\lambda_{i \tau} x_{4}+\lambda_{i \tau a} x_{5}+\lambda_{+} x_{6}\right) .
$$

If $\beta$ is taken as the bifurcation parameter and we consider $R_{0}^{a}=1$, then for $\beta=\beta^{* *}$ the Jacobian matrix for the DFE has zero as an eigenvalue, with the right eigenvector

$$
u=\left(u_{1}, u_{2}, u_{3}, u_{4}, u_{5}, u_{6}\right)^{T}
$$

and the left eigenvector

$$
v=\left(v_{1}, v_{2}, v_{3}, v_{4}, v_{5}, v_{6}\right)^{T},
$$


where

$$
\begin{gathered}
u_{1}=u_{1}>0, \\
u_{2}=\frac{c}{d+\theta_{1}} u_{1}, \\
u_{3}=u_{3}>0, \\
u_{4}=\frac{p \delta_{\tau}}{d+\mu_{I}+\nu+\sigma_{i \tau}} u_{3}, \\
u_{5}=\frac{(1-p) \delta_{\tau}\left(d+\mu_{I}+\nu+\sigma_{i \tau}\right)+\nu p \delta_{\tau}}{d+\mu_{I}+\nu+\sigma_{i \tau}} u_{3}, \\
u_{6}=\frac{\sigma_{i} u_{3}+\sigma_{i \tau} u_{4}+\sigma_{i \tau a} u_{5}}{d+\mu_{+}+\theta_{2}}
\end{gathered}
$$

and

$$
\begin{aligned}
v_{1}= & v_{1}>0, \\
v_{2}= & \frac{\theta_{1}}{d+\theta_{1}} v_{1}, \\
v_{3}= & \frac{d\left(d+c+\theta_{1}\right)}{d+\theta_{1}} v_{1}, \\
v_{4}= & \frac{1}{d+d_{I}+\theta_{2}}\left[\frac{d \theta_{2}\left(d+c+\theta_{1}\right)}{d+\theta_{1}}\right. \\
& \left.+\frac{\beta \lambda_{+}\left(d+\theta_{1}\right) r}{d+c+\theta_{1}}\right] v_{1}, \\
v_{5}= & \frac{1}{d+\mu_{A}+\sigma_{i \tau a}}\left[\frac{\beta \lambda_{i \tau a}\left(d+\theta_{1}\right) r}{d+d_{I}+\theta_{1}}\right. \\
& +\frac{\sigma_{i \tau a}}{d+d_{I}+\theta_{2}}\left[\frac{d \theta_{2}\left(d+c+\theta_{1}\right)}{d+\theta_{1}}\right. \\
& \left.\left.+\frac{\beta \lambda_{+} r}{d+c+\theta_{1}}\right]\right] v_{1}, \\
v_{6}= & \frac{1}{d+\mu_{I}+\nu+\sigma_{i \tau}}\left[\frac{\beta \lambda_{i \tau a}\left(d+\theta_{1}\right) r}{d+d_{I}+\theta_{1}} v_{1}\right. \\
& \left.+\nu v_{5}+\sigma_{i \tau} v_{6}\right],
\end{aligned}
$$

with

$$
r=\frac{d\left(d+c+\theta_{1}\right)}{d+\theta_{1}}-1 .
$$

Moreover, the bifurcation coefficient

$$
\begin{aligned}
b & =\sum_{k, i=1}^{6} v_{k} u_{i} \frac{\partial^{2} f_{k}(0,0)}{\partial x_{i} \partial \beta^{*}} \\
& =v_{3}\left[u_{3}+\lambda_{i \tau} u_{4}+\lambda_{i \tau a} u_{5}+\lambda_{+} u_{6}\right]
\end{aligned}
$$

is always positive, since $u_{3}, u_{4}, u_{5}, u_{6}, v_{3}>0$. It follows from the work of Bhunu et al. (2009) that when $\beta<\beta_{\text {critical }}$, there exists a unique stable disease-free equilibrium; and when $\beta$ changes from $\beta<\beta_{\text {critical }}$ to $\beta>$ $\beta_{\text {critical }}$, the disease-free equilibrium becomes unstable and the endemic equilibrium changes from negative to positive. This implies that the system (2) is locally asymptotically stable around the point $E^{* *}$. Thus we have the following result.

Theorem 10. For a sufficiently close $R_{0}^{a}>1$, the unique endemic equilibrium (EE), $E^{* *}$, is locally asymptotically stable.

4.2.2. Global stability of the $\mathbf{E E}\left(\boldsymbol{E}^{* *}\right)$. To prove the global stability of the endemic equilibrium, we assume that the population does not change significantly over the modelling time and the relation $\Pi=d N+d_{I} I+$ $\mu_{I} I_{D}+\mu_{A} I_{D A}+\mu_{+} I_{+}$holds. To attain the full characterization of endemic equilibrium, we construct a Lyapunov function and the results thus found are stated in the following theorem whose proof is given in Appendix B.

Theorem 11. The endemic equilibrium $E^{* *}$ is globally asymptotically stable if $\Pi=d N+d_{I} I+\mu_{I} I_{D}+$ $\mu_{A} I_{D A}+\mu_{+} I_{+}$and $R_{0}^{a}>1$.

4.3. Model persistence. Uniform persistence of system (2) implies that there exists a constant $\psi>$ 0 such that any solution starting with initial value $\left(S^{0}, S_{+}^{0}, I^{0}, I_{D}^{0}, I_{D A}^{0}, I_{+}^{0}\right)$ satisfies

$$
\begin{aligned}
\liminf _{t \rightarrow \infty} S(t) & \leq \psi, \\
\liminf _{t \rightarrow \infty} S_{+}(t) & \leq \psi, \\
\liminf _{t \rightarrow \infty} I(t) & \leq \psi, \\
\liminf _{t \rightarrow \infty} I_{D}(t) & \leq \psi, \\
\liminf _{t \rightarrow \infty} I_{D A}(t) & \leq \psi, \\
\liminf _{t \rightarrow \infty} I_{+}(t) & \leq \psi .
\end{aligned}
$$

Now, we construct the Lyapunov function following Korobeinikov and Maini (2004) as

$$
\begin{aligned}
\mathcal{L}\left(S, S_{+}\right. & \left., I, I_{D}, I_{D A}, I_{+}\right) \\
= & \omega_{1}\left(S-S^{* *} \ln S\right)+\omega_{2}\left(S_{+}-S_{+}^{* *} \ln S_{+}\right) \\
& +\omega_{3}\left(I-I^{* *} \ln I\right)+\omega_{4}\left(I_{D}-I_{D}^{* *} \ln I_{D}\right) \\
& +\omega_{5}\left(I_{D A}-I_{D A}^{* *} \ln I_{D A}\right) \\
& +\omega_{6}\left(I_{+}-I_{+}^{* *} \ln I_{+}\right) .
\end{aligned}
$$

The derivative of $\mathcal{L}$ along the solution path is given by

$$
\begin{aligned}
\mathcal{L}^{\prime}(S, & \left.S_{+}, I, I_{D}, I_{D A}, I_{+}\right) \\
= & \omega_{1}\left(S-S^{* *}\right) \frac{S^{\prime}}{S} \\
& +\omega_{2}\left(S_{+}-S_{+}^{* *}\right) \frac{S_{+}^{\prime}}{S_{+}}+\omega_{3}\left(I-I^{* *}\right) \frac{I^{\prime}}{I}
\end{aligned}
$$




$$
\begin{aligned}
& +\omega_{4}\left(I_{D}-I_{D}^{* *}\right) \frac{I_{D}^{\prime}}{I_{D}}+\omega_{5}\left(I_{D A}-I_{D A}^{* *}\right) \frac{I_{D A}^{\prime}}{I_{D A}} \\
& +\omega_{6}\left(I_{+}-I_{+}^{* *}\right) \frac{I_{+}^{\prime}}{I_{+}} \\
\leq & -\omega_{1}\left(S-S^{* *}\right)(\lambda+d+c)-\omega_{2}\left(S_{+}-S_{+}^{* *}\right) \\
& \times\left(d+\theta_{1}\right)-\omega_{3}\left(I-I^{* *}\right)\left(d+d_{I}+\delta_{\tau}+\sigma_{i}\right) \\
& -\omega_{4}\left(I_{D}-I_{D}^{* *}\right)\left(d+\mu_{I}+\nu+\sigma_{i \tau}\right) \\
& -\omega_{5}\left(I_{D A}-I_{D A}^{* *}\right)\left(d+\mu_{A}+\sigma_{i \tau a}\right) \\
& -\omega_{6}\left(I_{D A}-I_{D A}^{* *}\right)\left(d+\theta_{2}+\mu_{+}\right), \\
\leq & \chi\left[\left(S-S^{* *}\right)+\left(S_{+}-S_{+}^{* *}\right)+\left(I-I^{* *}\right)\right. \\
& \left.+\left(I_{D}-I_{D}^{* *}\right)+\left(I_{D A}-I_{D A}^{* *}\right)+\left(I_{D A}-I_{D A}^{* *}\right)\right],
\end{aligned}
$$

where $\chi$ is the minimum value of $\omega_{k}, k=1, \ldots, 6$. Therefore, $\mathcal{L}\left(S, S_{+}, I, I_{D}, I_{D A}, I_{+}\right)$is a Lyapunov function, as

$$
\mathcal{L}\left(S, S_{+}, I, I_{D}, I_{D A}, I_{+}\right)>0
$$

and

$$
\mathcal{L}^{\prime}\left(S, S_{+}, I, I_{D}, I_{D A}, I_{+}\right)=0
$$

at $E^{* *}$. Since $S(t), S_{+}(t), I(t), I_{D}(t), I_{D A}(t), I_{+}(t) \in$ $L^{1}$ are continuous and bounded with derivatives in $L^{\infty}$, we get

$$
\begin{aligned}
\left(S-S^{* *}\right) & +\left(S_{+}-S_{+}^{* *}\right)+\left(I-I^{* *}\right)+\left(I_{D}-I_{\tau}^{* *}\right) \\
& +\left(I_{D A}-I_{D A}^{* *}\right)+\left(I_{D A}-I_{D A}^{* *}\right) \rightarrow 0
\end{aligned}
$$

as $t \rightarrow \infty$ (Barbalat, 1959). Thus, from 24 we get

$$
\mathcal{L}^{\prime}\left(S, S_{+}, I, I_{D}, I_{D A}, I_{+}\right) \leq 0
$$

and we conclude from the Lyapunov-LaSalle properties that system (2) uniformly persists.

\section{Numerical simulations}

To study the dynamical behaviours of models (2) and (5), we perform numerical computations with the initial values $S(0)=150, S_{+}(0)=15, I(0)=$ $50, I_{D}(0)=30, I_{D A}(0)=20$ and $I_{+}(0)=10$. The set of parameter values is given in Table 1. These values are collected from different peer reviewed international journals, and the rest are hypothetical parameters relevant to HIV/AIDS. This set of parameter values is constant throughout the numerical experiments except the value of $\delta_{\tau}$ and $\sigma_{x \in E}, E=\{i, i \tau, i \tau a\}$. The modifying transmission parameters are given in Table 1, in which the values represent $\lambda_{j} \beta$ for $j \in$ $\{i \tau, i \tau a,+\}$. Numerical simulations have been performed using MATLAB (version 7.6.0). We will start by investigating the sensitivity of $R_{0}$ and $R_{0}^{a}$ for each parameter.
5.1. Sensitivity analysis. In this section, we use sensitivity analysis to investigate the impact of various intervention measures. By this method, we can identify the parameters that have high impact on the basic reproductive ratio, as well as on the HIV transmission. Here we derive the sensitivity index by using partial rank correlation coefficients (PRCCs) of the basic reproductive ratio with respect to the parameters. According to Abiodun et al. (2013), the normalized forward sensitivity index of $R_{0}$ with respect to a parameter $m$ is defined as follows:

$$
\Pi_{m}^{R_{0}}=\frac{\partial R_{0}}{\partial m} \times \frac{m}{R_{0}} .
$$

\begin{tabular}{|c|c|c|}
\hline Parameter & $\begin{array}{c}\text { Assigned value } \\
\left(\text { day }^{-1}\right)\end{array}$ & Reference \\
\hline$\Pi$ & 12 & $\begin{array}{c}\text { Samanta et al. (2014), } \\
\text { Misra et al. (2011) }\end{array}$ \\
\hline$\beta$ & 0.0025 & $\begin{array}{l}\text { Samanta et al. (2014), } \\
\text { Roy et al. (2015) }\end{array}$ \\
\hline$\lambda_{i \tau}$ & 0.0019 & Smith et al. (2010) \\
\hline$\lambda_{i \tau a}$ & 0.0015 & Smith et al. (2010) \\
\hline$\lambda_{+}$ & 0.0021 & Assumed \\
\hline$d$ & 0.005 & Hove et al. (2009) \\
\hline$d_{I}$ & 0.007 & Hove et al. (2009) \\
\hline$\mu_{I}$ & $4.713 \times 10^{-4}$ & Roy et al. (2015) \\
\hline$\mu_{A}$ & $8.712 \times 10^{-4}$ & Roy et al. (2015) \\
\hline$\mu_{+}$ & 0.007 & Roy et al. (2015) \\
\hline$\nu$ & $3.178 \times 10^{-4}$ & Roy et al. (2015) \\
\hline$\delta_{\tau}$ & $8.329 \times 10^{-4}$ & Roy et al. (2015) \\
\hline$c$ & 0.00125 & Roy et al. (2015) \\
\hline & & Misra et al. (2011) \\
\hline$p$ & 0.65 & Assumed \\
\hline$\sigma_{i}$ & 0.0015 & Roy et al. (2015) \\
\hline$\sigma_{i \tau}$ & 0.0022 & Assumed \\
\hline$\sigma_{i \tau a}$ & 0.0017 & Assumed \\
\hline$\theta_{1}$ & 0.0052 & Roy et al. (2015) \\
\hline & & Misra et al. (2011) \\
\hline$\theta_{2}$ & 0.0015 & Roy et al. (2015) \\
\hline
\end{tabular}

In Fig. 2, PRCCs are plotted for each input parameter for the model system (5). This demonstrates that $R_{0}$ is most negatively sensitive to the diagnosis and treatment rate, $\delta_{\tau}$, meaning that if we increase the diagnosis and treatment rate, that can reduce new cases and HIV prevalence. In Fig. 3, $R_{0}$ is drawn as a function of $\delta$ and $\tau$, which describes the significant dependence of $R_{0}$ on these parameters.

Figure 4(a) exhibits that $\delta_{\tau}$ and $\theta_{2}$ are two parameters that are negatively correlated with the reproductive number $R_{0}^{a}$ for system (2). A sharp decrease can be seen in the values of $R_{0}^{a}$ with variations in $\delta_{\tau}$ and $\theta_{2}$ (see Fig. 4(b)). This indicates that the model with treatment

Table 1. List of parameters used for the systems (2) and (5). 


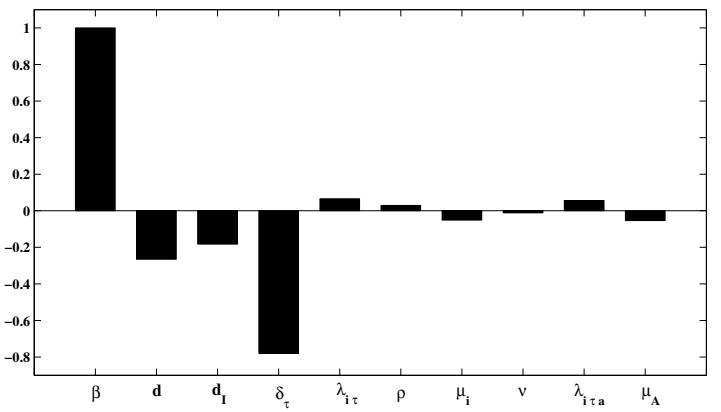

Fig. 2. Partial rank correlation coefficients indicate that the rate at which people are diagnosed and treated $\left(\delta_{\tau}=\delta \cdot \tau\right)$ is most sensitive to $R_{0}$. This means that the value of $R_{0}$ will decrease when $\delta$ and $\tau$ are increased.

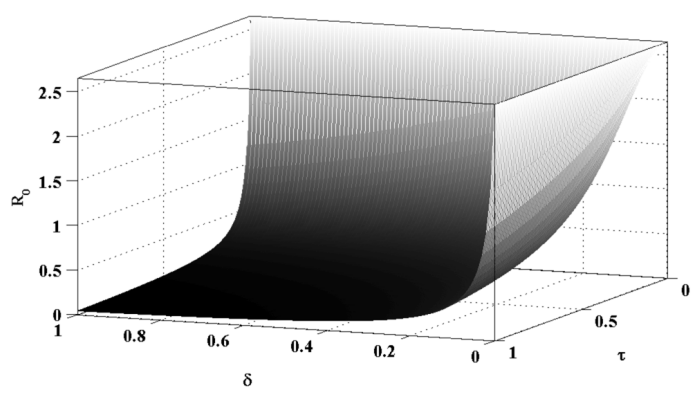

Fig. 3. Graph of $R_{0}$ as a function of $\delta$ and $\tau$.

and awareness is more feasible and has a more significant result than the model system without awareness.

5.2. Numerical results. We vary the population densities with respect to time (see Fig. 5), keeping all parameters fixed as same as in Table 1 except the values of $\sigma_{i}, \sigma_{i \tau}, \sigma_{i \tau a}, \theta_{1}$ and $\theta_{2}$. To show the advantage of awareness on the disease transmission that changes the epidemic trend, we vary the population densities with and without awareness in Fig. 5. Here, solid trajectories indicate the changes in population densities when treatment is only effective and dotted trajectories indicate the case when both treatment and awareness are included. Figure 5 indicates that after 500 days, the susceptible population $S(t)$ is increased by $20.85 \%$, while the infected population $(I(t))$, the screened but non-clinical AIDS population $\left(I_{D}(t)\right)$ and the population screened with clinical AIDS $\left(I_{D A}(t)\right)$ are decreased by $11.43 \%, 11.89 \%, 11.99 \%$, respectively. It is worth mentioning here that as we increase the values of $\sigma_{i}, \sigma_{i \tau}$ and $\sigma_{i \tau a}$, the percentage of all infected classes reduces. Since $R_{0}^{a}$ is a function of $\sigma_{i}, \sigma_{i \tau}$ and $\sigma_{i \tau a}$, the disease transmission rate can be controlled by varying these parameter values. However, the disease cannot be eradicated from the system, since the proper isolation of the disease transmission, i.e., $\beta=0$ from the infectious disease and/or the permanent immunity are not possible.

In Fig. 6, we plot the total susceptible population along the horizontal axis and the total infected population along the vertical axis for system (2). This figure shows the global stability around the endemic equilibrium point. For $\beta=0.00285$ and other fixed values of parameters as listed in Table 1, we obtain the interior equilibrium $E^{* *}(182,62)$. We choose different initial values

$$
(165,64),(170,55),(175,68),(180,54),
$$

$$
(185,62),(195,54),(200,60)
$$

and draw the phase portrait of system (2). We observed that all trajectories initiated from different initial values converge to the endemic equilibrium point $E^{* *}(182,62)$, which clearly indicates that the endemic equilibrium is globally asymptotically stable.

\section{Discussion and conclusions}

In this article, we deal with a non-linear mathematical model that incorporates the effect of drug-dosing along with the influence of media, where we measure the success of knowledge by its ability to reduce the risk of infection. We compare our model with a sub-model where it is assumed that no awareness programs are provided and only screening \& testing is available for the whole population. We have studied the impact of awareness as a novel intervention for HIV/AIDS control. In the modelling process, it is assumed that media campaigns create awareness regarding personal protection as well as control the disease spread. As a result, behavioural changes (transfer from unaware to aware) occur within the human population, which results in the formation of a new class, i.e., the aware class. Individuals of this class not only protect themselves from the infection, but being aware they also take part in reducing HIV/AIDS by taking precautions.

Our analytical study shows that the basic reproduction number, $R_{0}$, which determines the existence of the disease, contains awareness related parameters. As a result, the persistence of the disease depends on the rate of public consciousness. Awareness among people reduces the infection rate, shortens the rate of disease transmission and cuts down the size of the disease. Numerical simulations, which are very realistic, add an extra dimension to our analytic conclusions. Our results show that in presence of awareness in the population makes the disease transmission difficult and shorter. If awareness is provided along with treatment, then the HCT program will control the disease more rapidly. As a result, the best strategy is treating HIV/AIDS with proper 


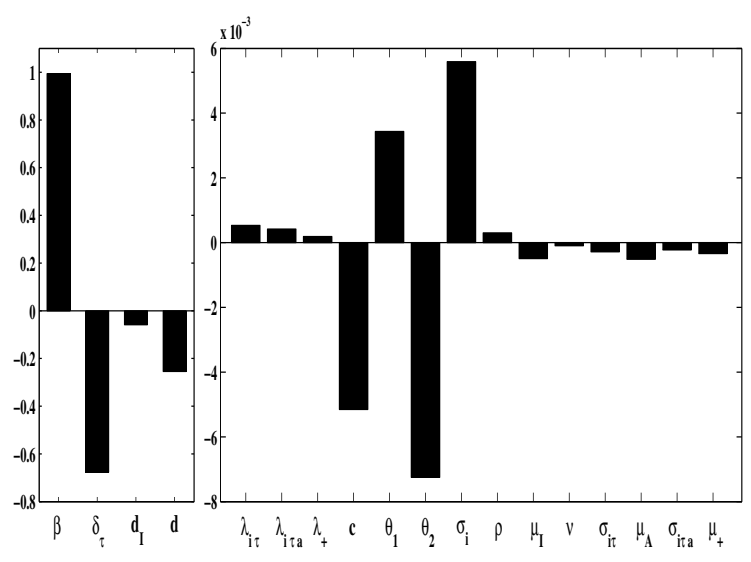

(a)

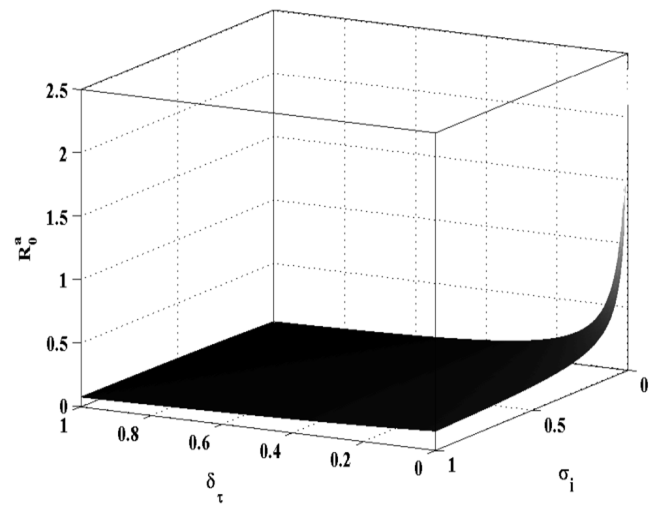

(b)

Fig. 4. Partial rank correlation coefficients indicate that the rate at which people are diagnosed and treated $\left(\delta_{\tau}\right)$ and the transfer rate from the unaware and untreated infected class to the aware infected class $\left(\sigma_{i}\right)$ are most negatively sensitive and have the greatest effect on $R_{0}^{a}$ (a). Graph of $R_{0}^{a}$ as a function of $\delta_{\tau}$ and $\sigma_{i}(\mathrm{~b})$.
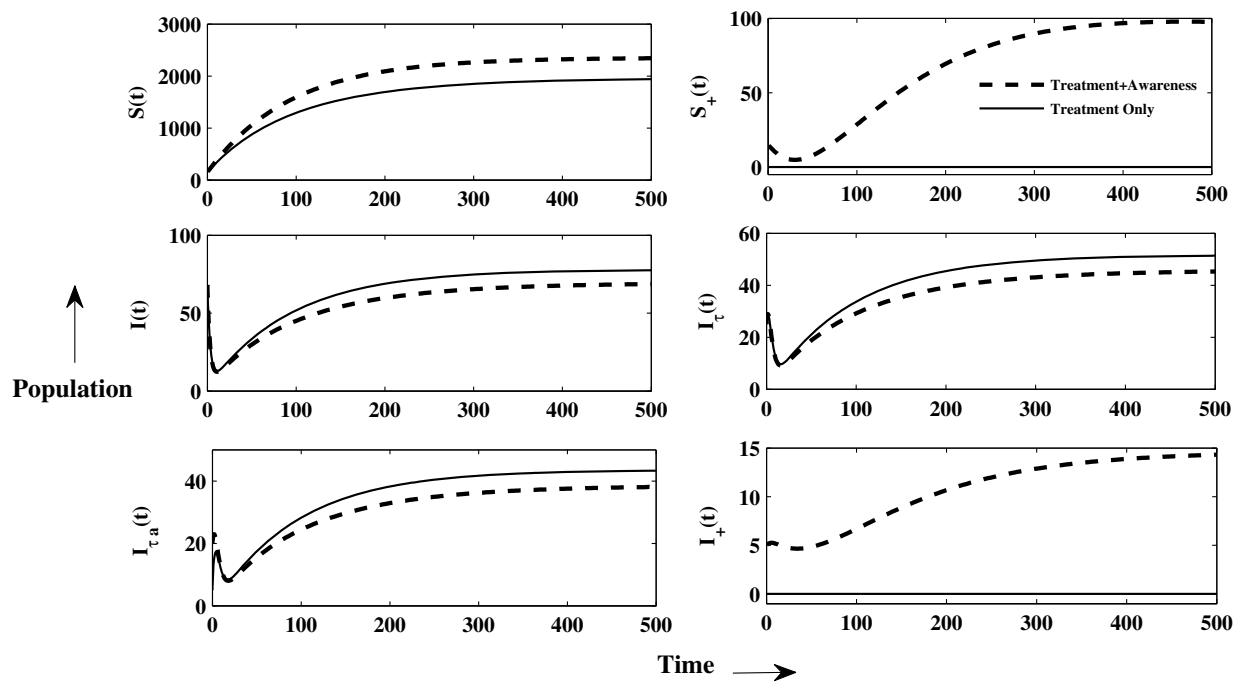

Fig. 5. Population densities are plotted with respect to time, where solid trajectories indicate the presence of drug without awareness and dotted trajectories indicate the presence of drug with awareness.

implementation of awareness programs through media. But in a practical sense, the disease remains endemic, because low education, ignorance in taking precautions, social problems, immigration, etc. play negative roles in the system. We discuss a model that captures some important features and we believe these findings may help in controlling AIDS through awareness.

\section{Acknowledgment}

The authors would like to thank the referees for their constructive and insightful comments which helped us to improve the quality of this work. The research presented here was funded by the Innovation in Science Pursuit for Inspired Research (INSPIRE) Program, Department of
Science and Technology, Government of India.

\section{References}

Abiodun, G.J., Marcus, N., Okosun, K.O. and Witbooi, P.J. (2013). A model for control of HIV/AIDS with parental care, International Journal of Biomathematics 6(02): 1350006

Al-arydah, M. and Smith, R. (2015). Adding education to test and treat: Can we overcome drug resistance?, Journal of Applied Mathematics 2015, Article ID 781270, DOI: 10.1155/2015/781270.

Barbalat, I. (1959). Systèmes d'équations diffèrentielles d'oscillations non-linéaires, Revue Roumaine de Mathématiques Pures et Appliquées 4(2): 267-270. 


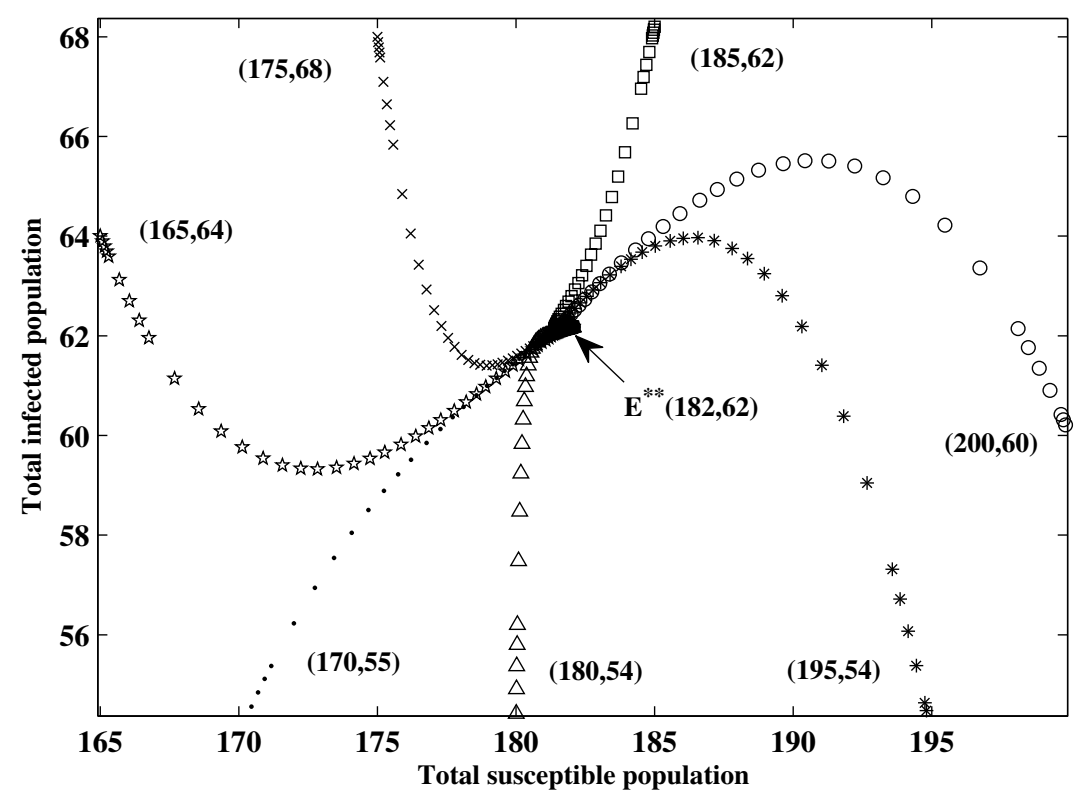

Fig. 6. Global stability of the endemic equilibrium for $\beta^{* *}=0.00285$; the other parameters are fixed as in Table 1 .

Bhunu, C., Garira, W. and Magombedze, G. (2009). Mathematical analysis of a two strain HIV/AIDS model with antiretroviral treatment, Acta Biotheoretica 57(3): 361-381.

Cai, L., Li, X., Ghosh, M. and Guo, B. (2009). Stability analysis of an HIV/AIDS epidemic model with treatment, Journal of Computational and Applied Mathematics 229(1): 313-323.

Castillo-Chavez, C., Blower, S., Driessche, P., Kirschner, D. and Yakubu, A.-A. (2002). Mathematical Approaches for Emerging and Reemerging Infectious Diseases: Models, Methods, and Theory, Springer, New York, NY.

Castillo-Chavez, C. and Song, B. (2004). Dynamical models of tuberculosis and their applications, Mathematical Biosciences and Engineering 1(2): 361-404.

Chatterjee, A.N. and Roy, P.K. (2012). Anti-viral drug treatment along with immune activator IL-2: A control-based mathematical approach for HIV infection, International Journal of Control 85(2): 220-237.

Chatterjee, A.N., Saha, S. and Roy, P.K. (2015). Human immunodeficiency virus/acquired immune deficiency syndrome: Using drug from mathematical perceptive, World Journal of Virology 4(4): 356.

Elbasha, E.H. and Gumel, A.B. (2006). Theoretical assessment of public health impact of imperfect prophylactic HIV-1 vaccines with therapeutic benefits, Bulletin of Mathematical Biology 68(3): 577.

Gumel, A.B., Castillo-Chavez, C., Mickens, R.E. and Clemence, D.P. (2006). Mathematical Studies on Human Disease Dy- namics: Emerging Paradigms and Challenges, Vol. 410, American Mathematical Society, Boston, MA.

Hove-Musekwa, S.D. and Nyababza, F. (2009). The dynamics of an HIV/AIDS model with screened disease carriers, Computational and Mathematical Methods in Medicine 10(4): 287-305.

Hyman, J.M., Li, J. and Stanley, E.A. (2003). Modeling the impact of random screening and contact tracing in reducing the spread of HIV, Mathematical Biosciences 181(1): $17-54$.

Kiss, I.Z., Cassell, J., Recker, M. and Simon, P.L. (2010). The impact of information transmission on epidemic outbreaks, Mathematical Biosciences 225(1): 1-10.

Korobeinikov, A. and Maini, P.K. (2004). A Lyapunov function and global properties for SIR and SEIR epidemiological models with nonlinear incidence, Mathematical Biosciences and Engineering 1(1): 57-60.

Misra, A., Sharma, A. and Singh, V. (2011). Effect of awareness programs in controlling the prevalence of an epidemic with time delay, Journal of Biological Systems 19(02): 389-402.

Nyabadza, F. (2006). A mathematical model for combating HIV/AIDS in southern Africa: Will multiple strategies work?, Journal of Biological Systems 14(03): 357-372.

Roy, P.K. (2015). Mathematical Models for Therapeutic Approaches to Control HIV Disease Transmission, Springer, Singapore.

Roy, P. K., Saha, S. and Al Basir, F. (2015). Effect of awareness programs in controlling the disease HIV/AIDS: An optimal control theoretic approach, Advances in Difference Equations 2015(1): 217-234. 
Samanta, S. and Chattopadhyay, J. (2014). Effect of awareness program in disease outbreak - a slow-fast dynamics, $A p$ plied Mathematics and Computation 237(8): 98-109.

Smith, R.J., Okano, J.T., Kahn, J.S., Bodine, E.N. and Blower, S. (2010). Evolutionary dynamics of complex networks of HIV drug-resistant strains: The case of San Francisco, Science 327(5966): 697-701.

Statistics (2006). Statistics of South Africa. Website of the mid-year population estimates, Statistical release P0302, South Africa, http://www.statssa.gov.za/ publications

Tripathi, A., Naresh, R. and Sharma, D. (2007). Modeling the effect of screening of unaware infectives on the spread of HIV infection, Applied Mathematics and Computation 184(2): 1053-1068.

UDAIDS/WHO (2014). Website of the UDAIDS/WHO epidemiological fact sheets on HIV and AIDS, http://www.who.int/hiv/en/

UDAIDS/WHO (2015). Website of the UDAIDS/WHO epidemiological fact sheets on HIV and AIDS, http://www. who.int/hiv/en/

Van den Driessche, P. and Watmough, J. (2002). Reproduction numbers and sub-threshold endemic equilibria for compartmental models of disease transmission, Mathematical Biosciences 180(1): 29-48.

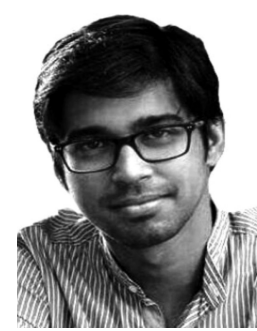

Shubhankar Saha was born in 1990 . He received the BSc and MSc degrees in mathematics with mathematical biology from Jadavpur University, Kolkata, India, in 2010 and 2012, respectively. Now he is a member of the Centre for Mathematical Biology and Ecology at the Department of Mathematics, Jadavpur University, and is pursuing his $\mathrm{PhD}$ from the same university. His research interests focus on the modeling, analysis and control of HIV dynamics.

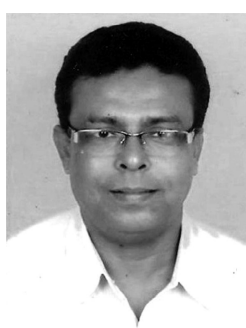

Priti Kumar Roy was born in 1967. He received his MSc degree in mathematics in 1991 and the $\mathrm{PhD}$ degree in applied mathematics in 2007 from Jadavpur University, Kolkata, India. He is currently a professor of applied mathematics of that university. His research is focused on modelling disease dynamics (like psoriasis and HIV), enzyme kinetics, bio-diesel production, etc.

\section{Appendix A}

\section{Proof of Theorem 7}

To study the global stability of the endemic equilibrium, we construct the Lyapunov function

$$
\begin{aligned}
V= & \left(S-S^{*}-S^{*} \ln \frac{S}{S^{*}}\right)+\phi_{1}\left(I-I^{*}-I^{*} \ln \frac{I}{I^{*}}\right) \\
& +\phi_{2}\left(I_{D}-I_{D}^{*}-I_{D}^{*} \ln \frac{I_{D}}{I_{D}^{*}}\right)+\phi_{3}\left(I_{D A}-I_{D A}^{*}\right. \\
& \left.-I_{D A}^{*} \ln \frac{I_{D A}}{I_{D A}^{*}}\right) .
\end{aligned}
$$

Its derivative is

$$
\begin{aligned}
V^{\prime}= & \left(1-\frac{S^{*}}{S}\right) \frac{\mathrm{d} S}{\mathrm{~d} t}+\phi_{1}\left(1-\frac{I^{*}}{I}\right) \frac{\mathrm{d} I}{\mathrm{~d} t} \\
& +\phi_{2}\left(1-\frac{I_{D}^{*}}{I_{D}}\right) \frac{\mathrm{d} I_{D}}{\mathrm{~d} t}+\phi_{3}\left(1-\frac{I_{D A}^{*}}{I_{D A}}\right) \frac{\mathrm{d} I_{D A}}{\mathrm{~d} t} .
\end{aligned}
$$

At the endemic equilibrium point, we have

$$
\begin{aligned}
\Pi & =\left(\lambda^{*}+d\right) S^{*}, \\
d+d_{I}+\delta_{\tau} & =\frac{\lambda^{*} S^{*}}{I^{*}}, \\
d+\mu_{I}+\nu & =\frac{p \delta_{\tau} I^{*}}{I_{D}^{*}}, \\
d+\mu_{A} & =\frac{(1-p) \delta_{\tau} I^{*}}{I_{D A}^{*}}+\frac{\nu I_{D}^{*}}{I_{D A}^{*}} .
\end{aligned}
$$

Now we let

$$
\frac{S}{S^{*}}=w, \quad \frac{I}{I^{*}}=x, \quad \frac{I_{D}}{I_{D}^{*}}=y \quad \frac{I_{D A}}{I_{D A}^{*}}=z
$$

and evaluate the components of the derivative of the Lyapunov function so that

$$
\begin{aligned}
(1- & \left.\frac{S^{*}}{S}\right) \frac{\mathrm{d} S}{\mathrm{~d} t} \\
= & -d \frac{S^{*}}{w}(1-w)^{2}+\varsigma\left(1-\frac{1}{w}\right)(1-w x) S^{*} I^{*} \\
& +\varsigma \lambda_{i \tau}\left(1-\frac{1}{w}\right)(1-w y) S^{*} I_{D}^{*} \\
& +\varsigma \lambda_{i \tau a}\left(1-\frac{1}{w}\right)(1-w z),
\end{aligned}
$$

$$
\begin{aligned}
\phi_{1}(1 & \left.-\frac{I^{*}}{I}\right) \frac{\mathrm{d} I}{\mathrm{~d} t} \\
= & \phi_{1} \varsigma\left(1-\frac{1}{x}\right)(w x-x) S^{*} I^{*} \\
& +\phi_{1} \varsigma \lambda_{i \tau}\left(1-\frac{1}{x}\right)(w y-x) S^{*} I_{D}^{*} \\
& +\phi_{1} \varsigma \lambda_{i \tau a}\left(1-\frac{1}{x}\right)(w z-x) S^{*} I_{D A}^{*},
\end{aligned}
$$

$$
\begin{aligned}
\phi_{2}\left(1-\frac{I_{D}^{*}}{I_{D}}\right) \frac{\mathrm{d} I_{D}}{\mathrm{~d} t} \\
=\phi_{2} p \delta_{\tau}\left(1-\frac{1}{y}\right)(x-y) I^{*}, \\
\phi_{3}\left(1-\frac{I_{D A}^{*}}{I_{D A}}\right) \frac{\mathrm{d} I_{D A}}{\mathrm{~d} t} \\
=\phi_{3}(1-p) \delta_{\tau}\left(1-\frac{1}{z}\right) \\
\quad(x-z) I^{*}+\phi_{3} \nu\left(1-\frac{1}{z}\right)(y-z) I^{*} .
\end{aligned}
$$


Substituting (A5)-A7 in A2 we get

$$
\frac{\mathrm{d} V}{\mathrm{~d} t}=-d \frac{S^{*}}{w}(1-w)^{2}+G(w, x, y, z),
$$

We choose

$$
\begin{gathered}
\phi_{1}=1, \quad \phi_{2}=\frac{\lambda_{i \tau} \varsigma S^{*} I_{D}^{*}}{p \delta_{\tau} I^{*}}, \\
\phi_{3}=\frac{\lambda_{i \tau a} \varsigma S^{*} I_{D A}^{*}}{(1-p) \delta_{\tau} I^{*}+\nu I_{D}^{*}}
\end{gathered}
$$

so that the expression for $G(w, x, y, z)$ in $(\mathrm{A} 8)$ becomes

$$
\begin{aligned}
G(w, x, y, z) & \\
= & \varsigma S^{*} I^{*}\left(2-\frac{1}{w}-w\right)+\varsigma \lambda_{i \tau} S^{*} I_{D}^{*}\left(2-\frac{1}{w}-\frac{w y}{x}\right) \\
& +\varsigma \lambda_{i \tau a} S^{*} I_{D A}^{*}\left(2-\frac{1}{w}-\frac{w z}{x}\right) \\
& -x \delta_{\tau}\left[\phi_{2} p \frac{I^{*}}{y}+\phi_{3}(1-p) \frac{I^{*}}{z}\right] \\
& -y \frac{\phi_{3} \nu I_{D}^{*}}{z}-z \phi_{3}\left[(1-p) \delta_{\tau} I^{*}+\nu I_{D}^{*}\right] .
\end{aligned}
$$

Note that the terms $(2-1 / w-w),(2-1 / w-w y / x)$ and $(2-1 / w-w z / x)$ are less than or equal to zero by the arithmetic mean-geometric mean inequality. This implies that $G \leq 0$ with equality only if $S=S^{*}, I=$ $I^{*}, I_{D}=I_{D}^{*}$ and $I_{D A}=I_{D A}^{*}$. Therefore, $\mathrm{d} V / \mathrm{d} t \leq 0$ and by LaSalle's extension, we can conclude that the point is globally asymptotically stable.

\section{Appendix B}

\section{Proof of Theorem 11}

We will use same approach as in Appendix A. To study the global stability of the endemic equilibrium for the system (2), we use the Lyapunov function

$$
\begin{aligned}
V_{a}= & \left(S-S^{*}-S^{*} \ln \frac{S}{S^{*}}\right)+\left(S_{+}-S_{+}^{*}-S_{+}^{*} \ln \frac{S_{+}}{S_{+}^{*}}\right) \\
& +\psi_{1}\left(I-I^{*}-I^{*} \ln \frac{I}{I^{*}}\right) \\
& +\psi_{2}\left(I_{D}-I_{D}^{*}-I_{D}^{*} \ln \frac{I_{D}}{I_{D}^{*}}\right) \\
& +\psi_{3}\left(I_{D A}-I_{D A}^{*}-I_{D A}^{*} \ln \frac{I_{D A}}{I_{D A}^{*}}\right) \\
& +\psi_{4}\left(I_{+}-I_{+}^{*}-I_{+}^{*} \ln \frac{I_{+}}{I_{+}^{*}}\right) .
\end{aligned}
$$

We set

$$
\frac{S_{+}}{S_{+}^{*}}=u, \quad \frac{I_{+}}{I_{+}^{*}}=v
$$

and the corresponding derivative of the Lyapunov function is given as

$$
\begin{aligned}
V_{a}^{\prime}= & \left(1-\frac{S^{*}}{S}\right) \frac{\mathrm{d} S}{\mathrm{~d} t}+\left(1-\frac{S_{+}^{*}}{S_{+}}\right) \frac{\mathrm{d} S_{+}}{\mathrm{d} t} \\
& +\psi_{1}\left(1-\frac{I^{*}}{I}\right) \frac{\mathrm{d} I}{\mathrm{~d} t}+\psi_{2}\left(1-\frac{I_{D}^{*}}{I_{D}}\right) \frac{\mathrm{d} I_{D}}{\mathrm{~d} t} \\
& +\psi_{3}\left(1-\frac{I_{D A}^{*}}{I_{D A}}\right) \frac{\mathrm{d} I_{D A}}{\mathrm{~d} t}+\psi_{4}\left(1-\frac{I_{+}^{*}}{I_{+}}\right) \frac{\mathrm{d} I_{+}}{\mathrm{d} t}
\end{aligned}
$$

where

$$
\begin{aligned}
&\left(1-\frac{S^{*}}{S}\right) \frac{\mathrm{d} S}{\mathrm{~d} t} \\
&=-(d+c) \frac{S^{*}}{w}(1-w)^{2}-\theta_{1} \frac{S_{+}^{*}}{u}(1-u)^{2} \\
&+\varsigma S^{*} I^{*}\left(1-\frac{1}{w}\right)(1-w x) \\
&+\varsigma \lambda_{i \tau} S^{*} I_{D}^{*}\left(1-\frac{1}{w}\right)(1-w y) \\
&+\varsigma \lambda_{i \tau a} S^{*} I_{D A}^{*}\left(1-\frac{1}{w}\right)(1-w z) \\
&+\varsigma \lambda_{+} S^{*} I_{+}^{*}\left(1-\frac{1}{w}\right)(1-w u), \\
&\left(1-\frac{S_{+}^{*}}{S_{+}}\right) \frac{\mathrm{d} S_{+}}{\mathrm{d} t}=c S^{*}\left(1-\frac{1}{u}\right)(w-u),
\end{aligned}
$$

$$
\begin{aligned}
\psi_{1}\left(1-\frac{I^{*}}{I}\right) \frac{\mathrm{d} I}{\mathrm{~d} t} \\
=\psi_{1} \theta_{1} \frac{I_{+}^{*}}{v}(1-v)^{2} \\
\quad+\psi_{1}\left(1-\frac{1}{x}\right)\left[\varsigma(w x-x) S^{*} I^{*}\right. \\
\quad+\varsigma \lambda_{i \tau}(w y-x) S^{*} I_{D}^{*}+\varsigma \lambda_{i \tau a}(w z-x) S^{*} I_{D A}^{*} \\
\left.\quad+\varsigma \lambda_{+}(w u-x) S^{*} I_{+}^{*}\right],
\end{aligned}
$$

$$
\psi_{2}\left(1-\frac{I_{D}^{*}}{I_{D}}\right) \frac{\mathrm{d} I_{D}}{\mathrm{~d} t}=\phi_{2} p \delta_{\tau}\left(1-\frac{1}{y}\right)(x-y) I^{*}
$$$$
\psi_{3}\left(1-\frac{I_{D A}^{*}}{I_{D A}}\right) \frac{\mathrm{d} I_{D A}}{\mathrm{~d} t}
$$$$
=\psi_{3}(1-p) \delta_{\tau}\left(1-\frac{1}{z}\right)(x-z) I^{*}
$$$$
+\psi_{3} \nu\left(1-\frac{1}{z}\right)(y-z) I^{*},
$$

$$
\begin{aligned}
\psi_{4} & \left(1-\frac{I_{+}^{*}}{I_{+}}\right) \frac{\mathrm{d} I_{+}}{\mathrm{d} t} \\
= & \psi_{4}\left(1-\frac{1}{v}\right)\left[\sigma_{i}(x-v) I^{*}+\sigma_{i \tau}(y-v) I_{D}^{*}\right. \\
& \left.+\sigma_{i \tau a}(z-v) I_{D A}\right] .
\end{aligned}
$$


Now, substituting (B3)- B8 in (B2), we get

$$
\begin{aligned}
V_{a}^{\prime}= & -(d+c) \frac{S^{*}}{w}(1-w)^{2}-\theta_{1} \frac{S_{+}^{*}}{u}(1-u)^{2} \\
& +\psi_{1} \theta_{1} \frac{I_{+}^{*}}{v}(1-v)^{2}+G_{a}(w, x, y, z, u, v),
\end{aligned}
$$

where

$$
\begin{aligned}
& G_{a}(w, x, y, z, u, v) \\
& \quad=G_{1 a}(w, x, y, z, u, v)+G_{2 a}(w, x, y, z, u, v) .
\end{aligned}
$$

We choose $\psi_{1}=1$ and the coefficients $\psi_{2}, \psi_{3}$ and $\psi_{4}$ can be obtained from the following expressions:

$$
\begin{aligned}
& \psi_{3} \nu I_{D}^{*}+\psi_{4} \sigma_{i \tau} I_{D}^{*}-\psi_{2} \rho \delta_{\tau} I^{*}=0, \\
& \psi_{4} \sigma_{i \tau a} I_{D A}^{*}-\left[(1-p) \delta_{\tau} I^{*}+\nu I_{D}^{*}\right] \psi_{3}=0, \\
& \psi_{2} \rho \delta_{\tau} I^{*}+\psi_{3}(1-p) \delta_{\tau} I^{*}+\psi_{4} \sigma_{i} I^{*} \\
& \quad-\varsigma \lambda_{i \tau} S^{*} I_{D}^{*}-\varsigma \lambda_{i \tau a} S^{*} I_{D A}^{*}-\varsigma \lambda_{+} S^{*} I_{+}^{*}=0,
\end{aligned}
$$

so that

$$
\begin{aligned}
& \psi_{2}=\frac{1}{\rho \delta_{\tau} I^{*}}\left(\frac{\nu \sigma_{i \tau a} I_{D A}^{*} I_{D}^{*}}{(1-p) \delta_{\tau} I^{*}+\nu I_{D}^{*}}+\sigma_{i \tau} I_{D}^{*}\right) \psi_{4}, \\
& \psi_{3}=\frac{\sigma_{i \tau a} I_{D A}^{*}}{(1-p) \delta_{\tau} I^{*}+\nu I_{D}^{*}} \psi_{4}, \\
& \psi_{4}=\frac{\varsigma\left(\lambda_{i \tau} S^{*} I_{D}^{*}+\lambda_{i \tau a} S^{*} I_{D A}^{*}+\lambda_{+} S^{*} I_{+}^{*}\right)}{\frac{\nu \sigma_{i \tau a} I_{D A}^{*} I_{D}^{*}}{(1-p) \delta_{\tau} I^{*}+\nu I_{D}^{*}}+\frac{(1-p) \delta_{\tau} \sigma_{i \tau a} I_{D A}^{*} I^{*}}{(1-p) \delta_{\tau} I^{*}+\nu I_{D}^{*}+\sigma_{i} I^{*}}+\sigma_{i \tau} I_{D}^{*}} .
\end{aligned}
$$

It is important to note that

$$
\begin{aligned}
& G_{1 a}(w, x, y, z, u, v) \\
& =2 \varsigma\left[S^{*} I^{*}+\lambda_{i \tau} S^{*} I_{D}^{*}+\lambda_{i \tau a} S^{*} I_{D A}^{*}+\lambda_{+} S^{*} I_{+}^{*}\right] \\
& \quad+\left[\psi_{2} \rho \delta_{\tau}+\psi_{3}(1-p) \delta_{\tau}+\psi_{4} \sigma_{i}\right] I^{*} \\
& \quad+\left(\psi_{3} \nu+\psi_{4} \sigma_{i \tau}\right) I_{D}^{*}+\psi_{4} \sigma_{i \tau a} I_{D A}^{*}+\varsigma \lambda_{+} S^{*} I_{+}^{*} u \\
& \quad+c S^{*} w+c S^{*}+\varsigma \lambda_{i \tau} S^{*} I^{*} y+\varsigma \lambda_{i \tau a} S^{*} I_{D A}^{*} z,
\end{aligned}
$$

$$
\begin{aligned}
G_{2 a} & (w, x, y, z, u, v) \\
= & -\varsigma S^{*} I^{*}\left(w+\frac{1}{w}\right)-\varsigma \lambda_{i \tau} S^{*} I_{D}^{*}\left(\frac{1}{w}+\frac{w y}{x}\right) \\
& -\varsigma \lambda_{i \tau a} S^{*} I_{D A}^{*}\left(\frac{1}{w}+\frac{w z}{x}\right)-\varsigma \lambda_{+} S^{*} I_{+}^{*}\left(\frac{1}{w}+\frac{w u}{x}\right) \\
& -c S^{*}\left(u+\frac{w}{u}\right)-\left(\psi_{2} \rho \delta_{\tau} \frac{x}{y}+\psi_{3}(1-p) \delta_{\tau} I^{*} \frac{x}{z}\right. \\
& \left.+\psi_{4} \sigma_{i} I^{*} \frac{x}{v}\right) I^{*}-\left(\psi_{3} \nu \frac{y}{z}+\psi_{4} \sigma_{i \tau} \frac{y}{v}\right) I_{D}^{*} \\
& -\psi_{4} \sigma_{i \tau a} \frac{z}{v} I_{D A}^{*}-\psi_{4}\left(\sigma_{i} I^{*}+\sigma_{i \tau} I_{D}^{*}+\sigma_{i \tau a} I_{D A}^{*}\right) v .
\end{aligned}
$$

Substituting the values of $\psi_{2}, \psi_{3}$ and $\psi_{4}$ in $G_{a}(w, x, y, z, u, v)$, we get

$$
\begin{aligned}
G_{a} & (w, x, y, z, u, v) \\
= & \varsigma S^{*} I^{*}\left(2-w-\frac{1}{w}\right)+\varsigma \lambda_{i \tau} S^{*} I_{D}^{*}\left(2-\frac{1}{w}-\frac{w y}{x}\right) \\
& +\varsigma \lambda_{i \tau a} S^{*} I_{D A}^{*}\left(2-\frac{1}{w}-\frac{w z}{x}\right) \\
& +\varsigma \lambda_{+} S^{*} I_{+}^{*}\left(2-\frac{1}{w}-\frac{w u}{x}\right) \\
& +G_{-}(w, x, y, z, u, v),
\end{aligned}
$$

where $G_{-}(w, x, y, z, u, v) \quad<\quad 0 . \quad$ Therefore, $G_{a}(w, x, y, z, u, v)$ is less than or equal to zero by the arithmetic mean-geometric mean inequality, which implies $G_{a} \leq 0$ with equality only if $S=S^{*}, S_{+}=S_{+}^{*}, I=I^{*}, I_{D}=I_{D}^{*}, I_{D A}=I_{D A}^{*}$ and $I_{+}=I_{+}^{*}$. Hence we prove that the endemic equilibrium point for the system (2) is globally asymptotically stable by LaSalle's extension.

Received: 30 June 2016 Revised: 24 November 2016 Re-revised: 14 January 2017 Accepted: 16 January 2017 